\title{
Statistics of Reconnecting Current Sheets in the Transition Region of Earth's Bow Shock
}

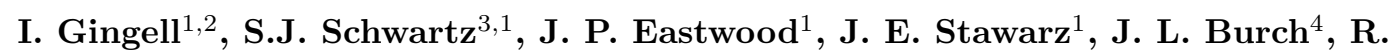 \\ E. $\operatorname{Ergun}^{3}$, S. A. Fuselier ${ }^{4}$, D. J. Gershman ${ }^{5}$, B. L. Giles ${ }^{5}$, Y. V. \\ Khotyaintsev $^{6}$, B. Lavraud ${ }^{7}$, P.-A. Lindqvist ${ }^{6}$, W. R. Paterson ${ }^{5}$, T. D. Phan $^{8}$, \\ C. T. Russell ${ }^{9}$, R. J. Strangeway ${ }^{9}$, R. B. Torbert $^{10}$, F. Wilder ${ }^{3}$ \\ ${ }^{1}$ The Blackett Laboratory, Imperial College London, SW7 2AZ, United Kingdom \\ ${ }^{2}$ School of Physics and Astronomy, University of Southampton, SO17 1BJ, United Kingdom \\ ${ }^{3}$ Laboratory for Atmospheric and Space Physics, University of Colorado, Boulder, Colorado 80303, USA \\ ${ }^{4}$ Southwest Research Institute, San Antonio, Texas 78238, USA \\ ${ }^{5}$ NASA, Goddard Space Flight Center, Greenbelt, Maryland 20771, USA \\ ${ }^{6}$ Swedish Institute of Space Physics (Uppsala), Uppsala, Sweden \\ ${ }^{7}$ Institut de Recherche en Astrophysique et Planétologie, CNRS, UPS, CNES, Université de Toulouse, \\ Toulouse, France \\ ${ }^{8}$ Space Science Laboratory,University of California, Berkeley, California, USA \\ ${ }^{9}$ University of California, Los Angeles, Los Angeles, California 90095, USA \\ ${ }^{10}$ University of New Hampshire, Durham, New Hampshire 03824, USA
}

\section{Key Points:}

- A survey of MMS observations of Earth's bow shock shows that reconnection is often present within the transition region.

- Current sheets are localised to the shock transition region, separate from magnetosheath turbulence further downstream.

- The primary consequence of reconnection in shocks is on magnetic topology, rather than heating.

Corresponding author: Imogen Gingell, i.l.gingell@soton.ac.uk 


\section{Abstract}

We have conducted a comprehensive survey of burst mode observations of Earth's bow shock by the Magnetospheric Multiscale (MMS) mission to identify and characterise current sheets associated with collisionless shocks, with a focus on those containing fast electron outflows, a likely signature of magnetic reconnection. The survey demonstrates that these thin current sheets are observed within the transition region of approximately $40 \%$ of shocks within the burst mode dataset of MMS. With only small apparent bias towards quasi-parallel shock orientations and high Alfvén Mach numbers, the results suggest that reconnection at shocks is a universal process, occurring across all shock orientations and Mach numbers. On examining the distributions of current sheet properties, we find no correlation between distance from the shock, sheet width or electron jet speed, though the relationship between electron and ion jet speed supports expectations of electrononly reconnection in the region. Furthermore, we find that robust heating statistics are not separable from background fluctuations, and thus the primary consequence of reconnection at shocks is in relaxing the topology of the disordered magnetic field in the transition region.

\section{Introduction}

Collisionless shocks are ubiquitous across astrophysical and space plasma environments, including planetary and stellar bow shocks, interplanetary shocks in the solar wind, and supernova remnants (Burgess \& Scholer, 2015). In reducing flows from super- to subsonic speeds, shocks in these environments must dissipate energy by "kinetic" plasma processes involving direct interaction of the ions and electrons with the electromagnetic fields. Understanding which microphysical processes are at play, and how, is critical for characterising particle heating and acceleration at shocks (Auer, Hurwitz, \& Kilb, 1962; Gosling \& Robson, 1985; Morse, Destler, \& Auer, 1972). However, these phenomena are strongly dependent on shock parameters such as the Alfvén Mach number $\left(M_{A}\right)$, plasma beta $(\beta)$, and the angle between the upstream magnetic field and shock normal $\left(\theta_{B n}\right)$ (Burgess \& Scholer, 2015).

Recent simulations of quasi-parallel shocks $\left(\theta_{B n}<45^{\circ}\right)$ (Gingell et al., 2017) and perpendicular shocks $\left(\theta_{B n}=90^{\circ}\right)($ Bohdan, Niemiec, Kobzar, \& Pohl, 2017; Matsumoto, Amano, Kato, \& Hoshino, 2015) have shown that kinetic processes occurring within the shock foot can generate current sheets and magnetic islands. In these simulations, cur- 
rent sheets and magnetic islands undergo magnetic reconnection, for which localised changes in magnetic topology result in rapid transfer of energy from fields to particles.

In the standard model, typical of large-scale current sheets at the magnetopause, reconnection occurs within an electron-scale diffusion region (Burch et al., 2016; Vasyliunas, 1975), while at ion scales coupled ions are ejected from the diffusion region as bidirectional jets (Gosling, Skoug, McComas, \& Smith, 2005; Paschmann et al., 1979; Phan et al., 2000). Reconnection exhausts then extend to much larger scales. In turbulent plasmas such as the magnetosheath or solar wind, magnetic reconnection is thought to play an important role in dissipation of energy at kinetic scales (Chasapis et al., 2018; Matthaeus \& Lamkin, 1986; Retinò et al., 2007; Servidio, Matthaeus, Shay, Cassak, \& Dmitruk, 2009; Sundkvist, Retinò, Vaivads, \& Bale, 2007; Yordanova et al., 2016). In the case of turbulent reconnection, observations by Phan et al. (2018) have shown that in the magnetosheath, reconnecting current sheets may not exhibit an ion exhaust at ion-scales or larger. Instead, the electron diffusion region encompasses the entire thin current sheet. This observation contrasts with others in the magnetosheath, for which ion exhausts have been observed (Eastwood et al., 2018; Øieroset et al., 2017; Vörös et al., 2017).

In the case presented by Gingell et al. (2017), the generation of reconnecting current sheets at a quasi-parallel shock was modulated by a cyclic self-reformation of the shock ramp, driven by reflected and back-streaming ions (Biskamp \& Welter, 1972; Burgess, 1989, 1995; Hada, Oonishi, Lembège, \& Savoini, 2003; Krauss-Varban \& Omidi, 1991; Scholer, Shinohara, \& Matsukiyo, 2003). In combination, these kinetic processes lead to the formation of a distinct turbulent or disordered transition region close to the shock ramp, separating the solar wind from the magnetosheath proper. For the purposes of this study, the shock transition region encompasses the region over which shock driven processes generate structure and fluctuations both upstream and downstream of the shock ramp. This includes upstream structures associated with back-streaming and reflected ions (i.e. the foot), the shock ramp, the overshoot and undershoot and similar large amplitude downstream fluctuations preceding the relatively quiescent magnetosheath. Within the transition region generated in the simulations by Gingell et al. (2017), magnetic islands merge by reconnection to form larger scale structures that are convected downstream. 
Recent observations of Earth's bow shock by the Magnetospheric Multiscale mission have confirmed that active reconnection is indeed occurring within the shock's transition region (Gingell et al., 2019; Wang et al., 2019), which extends from the shock foot and downstream of the shock ramp. Although these observations established the occurrence of reconnection at shocks, further open questions remain. For example, although an encounter with an ion exhaust has been described by Wang et al. (2019), many of the structures observed to date only exhibit evidence of coupling to the electrons. For that subset of events, there are no associated ion outflow jets or coincident increases in the ion temperature. Observations of reconnection further downstream in the magnetosheath also show electron-only reconnection (Phan et al., 2018), thus raising the question of how reconnection at the shock is linked to similar turbulent reconnection processes in the magnetosheath. However, for the cited shock observations, the current sheet widths are at ion scales rather than electron scales. Second, the shock reconnection case studies do not establish the frequency of this phenomenon, nor therefore its impact on energy re-partition at shocks. In the observations, the lack of ion response in some cases confirms that a hybrid particle-in-cell model cannot fully capture the energetics of these structures.

Given recent case studies of reconnection at the shock in both simulations and observations, we must next asses the integrated impact of reconnection on shock dynamics and energetics by adopting a statistical approach to the analysis of spacecraft observations. In this paper, we present a survey of current sheets exhibiting electron outflows (i.e. active reconnection sites) at Earth's bow shock, observed during Phase 1 of the Magnetospheric Multiscale mission (Burch et al., 2016). We examine the frequency of observation of shock waves exhibiting reconnection, the parameters of those shocks, and the statistics of the properties of the reconnecting current sheets. The survey is therefore able to target the following key questions: i) Which shock parameters and geometries lead to the generation of reconnecting current sheets? ii) Where does reconnection occur relative to the shock ramp? iii) What are the distributions of current sheet sizes and jet speeds, and how does that relate to the frequency of electron-only reconnection? iv) Do current sheets at the shock generate measurable heating signatures? We find that quasi-parallel and high-Mach number shocks generate more current sheets, that reconnection at shocks is separable from the population of reconnection sites associated with turbulence of the magnetosheath, and that reconnecting current sheets are more common in the downstream transition region than the foot. Furthermore, we show that cur- 
rent sheet properties such as width and jet speed are uncorrelated, and that any ion response is typically much weaker than the electron response, supporting an electron-only reconnection model. Given that the temperature response is weak for both ions and electrons, we finally conclude that the energy released by reconnection is not often observable as heating local to the reconnection site. Thus we expect that the primary consequence of reconnection in the shock transition region is in relaxing the magnetic fluctuations generated in the shock foot and ramp.

\section{Survey Method}

The following survey is performed for all bow shock crossings during the period 7th October 2015 to 9th February 2017 for which all necessary burst data are available for all four MMS spacecraft. The survey period corresponds to MMS mission phases 1A and 1B. Within that period, 223 shock crossings are available with sufficient burst data to conduct the following analysis. Electromagnetic field data are provided by the flux gate magnetometer (FGM) (Russell et al., 2016) and electric field double probe (EDP) (Lindqvist et al., 2016), both within the FIELDS suite (Torbert et al., 2016). Particle data have been provided by the Fast Plasma Investigation (FPI) (Pollock et al., 2016). The sampling frequency is $128 \mathrm{~Hz}$ for the FGM magnetic fields, and $8 \mathrm{kHz}$ for the EDP electric fields. The full three-dimensional ion phase space is sampled by FPI every $0.15 \mathrm{~s}$, and the electron phase space is sampled every $0.03 \mathrm{~s}$.

For each burst interval containing a shock, the shock parameters are determined by the following method:

1. The times at which the spacecraft MMS1 crosses the shock ramp and the boundary between the transition region and magnetosheath (if apparent) are chosen manually by inspection of the magnetic field and particle moments. Time $t_{s h}$ corresponds to the shock ramp, i.e. the boundary between the solar wind and shock transition region which extends downstream. Time $t_{t r}$ corresponds to the boundary between the shock transition region and the magnetosheath.

2. Upstream, downstream and transition region plasma parameters are then determined using the mean of the fields and moments in the intervals upstream of $t_{s h}$, downstream of $t_{t r}$, and between $t_{s h}$ and $t_{t r}$ respectively. 
3. The shock normal $\hat{\mathbf{n}}_{s h}$, shock speed $v_{s h}$, and orientation $\theta_{B n}$ are determined by three separate methods: i) performing a four-spacecraft timing analysis (Schwartz, 1998) on the electron number density time series, across a 4 s interval centred on the shock ramp time $t_{s h}$;ii) using the Peredo shock model (Peredo, Slavin, Mazur, \& Curtis, 1995) given the upstream solar wind conditions for the interval upstream of $t_{s h}$, and scaled to the position of MMS1; and iii) using the magnetic field and electron bulk velocities upstream and downstream of the shock, given the requirements set by co-planarity theorem (Abraham-Shrauner, 1972; Schwartz, 1998).

4. The Alfvén Mach number $M_{A}$ is derived for each interval from the mean fields and electron bulk plasma parameters for the period upstream of the shock ramp, i.e. $M_{A}=\mathbf{v}_{e} \cdot \hat{\mathbf{n}}_{s h} / v_{\mathrm{A}, \text { upstream }}$.

Within each burst interval containing a bow shock crossing, candidate reconnection sites are identified by the following method:

1. A time series of the current density is obtained from the curl of the magnetic field, using the magnetic field data from FGM for the four MMS spacecraft (Robert, Dunlop, Roux, \& Chanteur, 1998).

2. The algorithm identifies time intervals for which the magnitude of the current density exceeds three times the standard deviation calculated from the full burst interval. A Gaussian filter of width $0.08 \mathrm{~s}$ is applied to the time series of the magnitude of the current density prior to this test in order to ensure that regions of strong currents are not split within a given structure.

3. Each contiguous time interval for which $|J|>3 \sigma_{J}$ is uniquely labelled. Within each of those intervals, the maximum of $|J|$ and corresponding half-maxima are identified. The interval between bounding half-maxima of the peak in $|J|$ is considered the current-carrying region.

4. A coordinate system for each strong current interval is found using minimum variance analysis (Gosling \& Phan, 2013; Phan et al., 2018), identifying the maximum $(L)$, intermediate $(M)$ and minimum $(N)$ variance directions of the magnetic field from the FGM magnetic field data over the current-carrying region.

5. The algorithm then identifies an event as a candidate active reconnection site if the following conditions are met: i) the sign of the maximum variance component of the magnetic field $B_{L}$ changes sign across the current carrying region, ii) there 
is a peak in the $L$-component of the electron bulk velocity $V_{e L}$ within the current carrying region that deviates from the mean by more than one standard deviation. That deviation of the bulk velocity is expected to correspond to an electron outflow jet.

Finally, we determine the properties of each candidate reconnection event identified by the survey algorithm as discussed in each relevant section. Following the automated survey, a manual inspection of each candidate event was performed eliminate false or ambiguous identifications. In order to be considered a positive observation, a given candidate must display the following features: i) it is not part of a periodic structure, such as a wave; ii) there is a significant peak in the electron bulk velocity in the maximum variance direction $V_{e L}$, which is within the bounds of the magnetic field reversal and well distinguished from fluctuations outside the sheet; iii) current density is predominantly in the intermediate variance $M$-direction; and iv) the $L$-component of the magnetic field $\delta B_{L}$ and electron bulk velocity $\delta V_{e L} \sqrt{\mu_{0} m_{p} n}$ are not similarly correlated across the current carrying region. We note that criteria (iv) is line with the Walen test, for which we expect to see a change in sign of the correlation across the field reversal. However, a strict change in sign may not be observed if the electron outflow is offset (as may be the case for asymmetric or guide field reconnection. In ambiguous cases, we may also examine the eigenvalues of the coordinate transform matrix generated by the minimum variance analysis, in order to ensure that the minimum and intermediate variance directions are not degenerate. A poor quality minimum variance analysis in this regard indicates that an observed structure is not quasi-1D, i.e. it is not sheet-like.

An example of a reconnecting current sheet identified by the survey is shown in Figure 1 . In this case, the field reversal is observed approximately 30 s before the spacecraft crosses the shock ramp from the magnetosheath into the solar wind. The shock orientation (determined using a shock model) is $\theta_{B n} \sim 85^{\circ}$, and $M_{A} \sim 2$. Panel (k) demonstrates that the correlation between the $L$ components of the magnetic field and electron velocity reverses across the electron jet, satisfying the Walen test for the observation of active reconnection (Gosling et al., 2005). Panel (i) demonstrates that there is a peak in the electron temperature coincident with the current sheet, suggesting that the plasma is heating as a result of reconnection. 

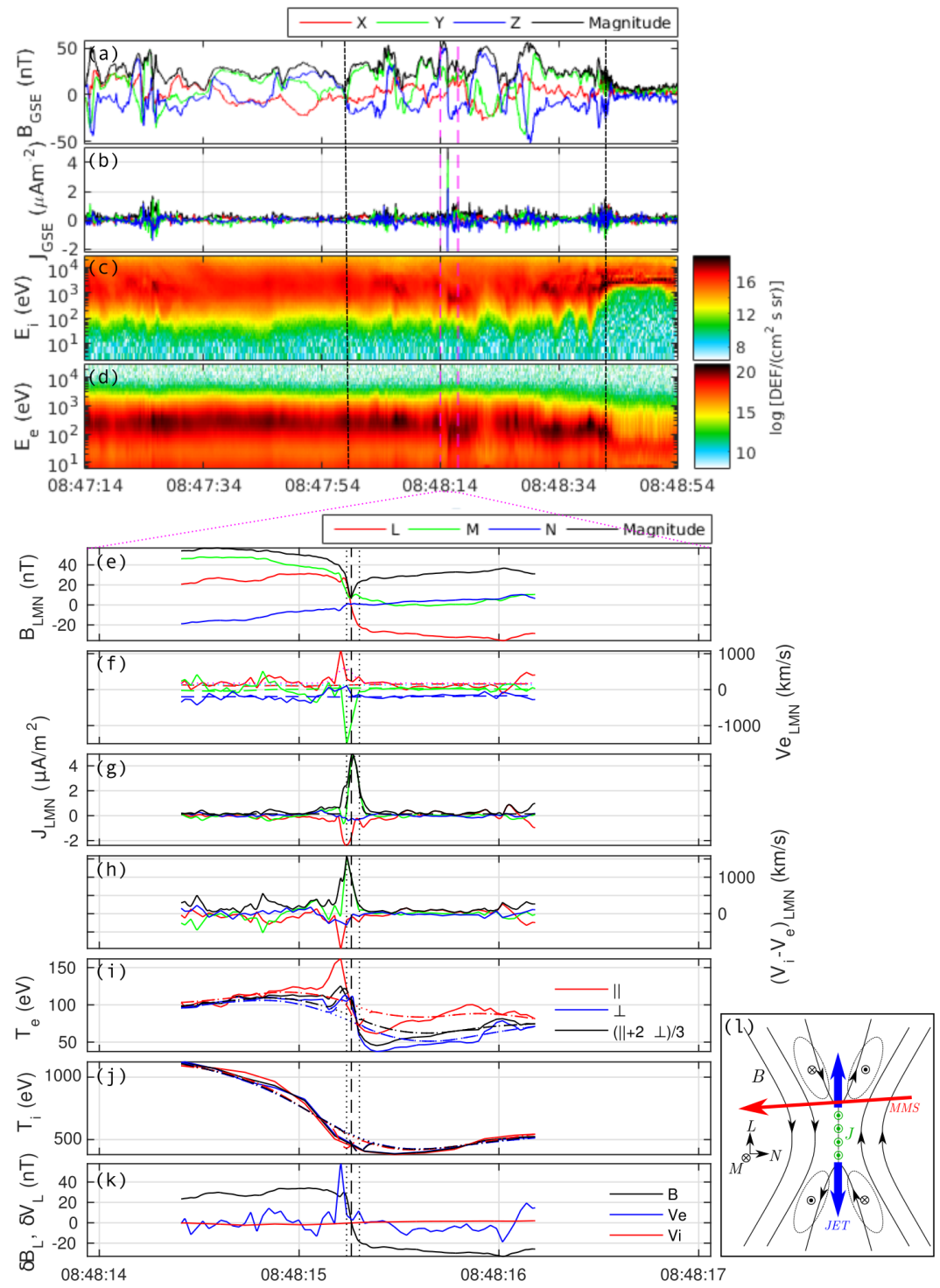

Figure 1. An example of an active reconnection site identified close to the bow shock crossing observed by MMS1 on 23rd December 2016 at 08:48:40UTC. Panels (a)-(d), for the full burst interval: magnetic field in GSE coordinates; current density in GSE coordinates; spectrogram of the ion differential energy flux; spectrogram of the electron differential energy flux. Dashed black lines show the locations of the shock ramp and edge of the transition region, and dashed magenta lines show the boundaries of the example interval. Panels (e)-(k), showing a close-up of an automatically identified reconnection site: magnetic field in minimum variance coordinates LMN, bulk electron (solid) and ion (dashed) velocities; current density from the curl of the magnetic field; the difference between ion and electron bulk velocities; electron temperature; ion temperature; magnetic field fluctuations $\delta B_{L}$ and velocity fluctuations $\delta v_{L} / \sqrt{\mu_{0} \rho}$ over-plotted to highlight the location of outflows with respect to field reversals. Panel (l): the trajectory of the MMS spacecraft through the example current sheet. 


\section{Results}

The automated survey identified 904 candidate reconnection events within the available shock crossings. Of the potential observations, the manual search identified 212 as current sheets. However, 47 of those structures did not show clear evidence of active reconnection, i.e. there we no significant electron or ion jets. Thus, the survey identified 165 actively reconnecting current sheets. These reconnecting current sheets were observed at 90 shocks out of the 223 shock crossings included in the survey. Hence, reconnection is captured by MMS at $40 \%$ of shocks observed during Phase 1 of the mission.

The full list of 165 active reconnection events is given in the Supplemental Material, each with associated shock parameters and current sheet properties.

\subsection{Shock Parameters}

Given that reconnection has only been observed within $40 \%$ of shock crossings during Phase 1A of the MMS mission, it is important to quantify the kinds of shocks that can generate active reconnection sites within the transition region. The distributions of key shock parameters $\theta_{B n}$ and $M_{A}$ are shown in Figure 2. The distribution of shock orientation $\theta_{B n}$ is shown for all three methods calculated within the survey: co-planarity theorem, timing analysis and the Peredo shock model (see section 2).

We note that different methods of determining the shock orientation $\theta_{B n}$ produce significantly different distributions. Since non-stationary and non-planar structure within the shock ramp such as ripples and shock reformation (Gingell et al., 2017) can cause local, ion-scale deviations in the shock orientation, timing analysis is unlikely to properly capture the global orientation of the shock for quasi-parallel shocks. Hence, we consider the shock model method (third column in Figure 2) to be most reliable. We define the distribution function $P_{\text {all }}(x)$ as the probability of observing a given parameter $x$ across all 223 shocks included in the survey. Likewise, we define the distribution function $P_{\text {rec }}(x)$ as the probability of observing a given parameter $x$ across only those 90 shocks for which at least one current sheet with an electron outflow was observed. The probability distribution $P_{\text {all }}\left(\theta_{B n, \text { model }}\right)$ in this case demonstrates that parallel shocks are less commonly observed by MMS than quasi-perpendicular shocks. This is expected given that intervals containing parallel shocks are more difficult to identify as clear, thin bound- 
ary layers, and are thus less likely to be selected for downlink during the data selection process.

The bottom row of Figure 2 shows a ratio of the probability distribution of shock parameters for all observed shocks and for those exhibiting signatures of reconnection, $P_{\text {rec }} / P_{\text {all }}$. For $P_{\text {rec }} / P_{\text {all }}>1$, for example, a given parameter range is more common within the population of shocks exhibiting current sheets with electron outflows. We find that $P_{\text {rec }} / P_{\text {all }} \approx 1$ across all parameter ranges within the given errors, for both $\theta_{B n}$ and $M_{A}$. This suggests that reconnection within the shock transition layer is a universal process. However, from the distributions of $\theta_{B n, \text { timing }}, \theta_{B n \text {,model }}$ and $M_{A}$, we observe small biases towards quasi-parallel and high Mach number shocks. This suggests that non-stationary processes and instabilities, observed more frequently at quasi-parallel and/or high Mach number shocks, may lead to the generation of more current sheets, but uniquely quasiparallel shock phenomena cannot be solely responsible for the occurrence of conditions conducive for reconnection.

\subsection{Sheet Locations}

On examining the location of each identified reconnection site with respect to geocentric solar ecliptic (GSE) coordinates, we find (as expected) that the current sheets are observed in a band approximately $10-12 R_{E}$ resembling the bow shock geometry, restricted by the orbits of MMS during Phase 1A. The spatial distribution is not shown in this paper, though the data are included in the supplemental material.

A histogram of the distribution of reconnection sites as a function of the time $t_{s h}$ between sheet observation and MMS1 crossing the bow shock ramp is shown in the left column of Figure 3, where $t_{s h}<0$ corresponds to the upstream solar wind region. Likewise, the distribution of a "pseudo-distance" of a reconnection event from the shock ramp, given by $D_{s h}=v_{s h} t_{s h}$, is shown in the right column. The shock velocity $v_{s h}$ is determined by timing analysis on the shock ramp at $t_{s h}$, as discussed in Section 3.1. Hence, for this section only, the dataset is down-sampled to include only those shocks for which the timing analysis returned valid (non-infinite) solutions. Furthermore, we note that there are significant errors associated with the pseudo-distance measure $D_{s h}$ due to the assumption of a constant shock speed across the spacecraft, $v_{s h}$. Owing to the dynamic nature of the system, the shock is not expected to continue to propagate at the same speed 

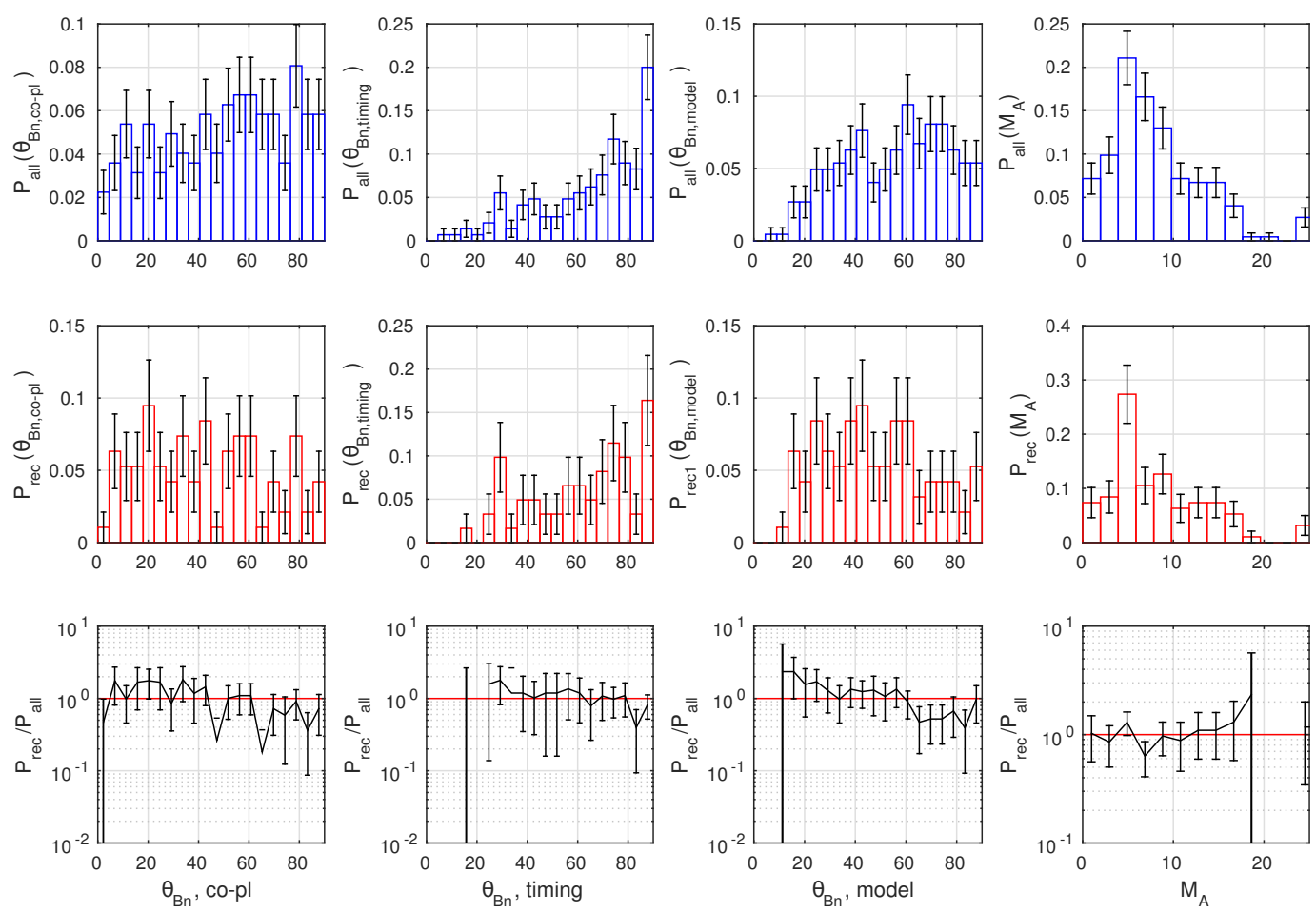

Figure 2. Top row: histograms showing the probability across all 223 shocks in the survey of observing a given shock orientation $\theta_{B n}$, calculated using co-planarity theorem (left), timing analysis (middle-left) and shock model (middle-right), along with Alfvén Mach number (right). Middle row: histograms showing the probability of observing a given orientation or Mach number for the 90 shocks at which at least one reconnecting current sheet was observed. Bottom row: Ratio of the probabilities of orientation and Mach number for shocks exhibiting reconnection and all shocks in the survey. The error bars represent a $\sqrt{N}$ error, where $N$ is the number of shocks recorded within each bin. 
for the full length of a given burst interval. Hence, the error increases significantly with time, and pseudo-distance $D_{s h}$ is likely to be a significant overestimate of the true distance of an event from the shock ramp.

The histograms demonstrate that the population of reconnection events is well localised to the shock ramp, within $50 \mathrm{~s}$ or $\sim 5 R_{E}$. However, in order to remove selection biases, we must consider how long the spacecraft observed any given region of space. This is especially important since the data selection process biases towards burst modes that contain thin, easily-identifiable boundaries. The probabilities $P\left(t_{s h}\right)$ and $P\left(D_{s h}\right)$ can then be weighted by the corresponding "dwell time" $t_{\mathrm{dwell}}$ to provide a metric of how common reconnection sites are at any given location. The dwell time is calculated by generating a histogram of time (or pseudo-distance) from the shock for every burst mode interval included in the survey, multiplied by the interval width of each bin in the histogram. The weighted distribution $P\left(D_{s h}\right) / t_{\text {dwell }}$ (Figure 3 , bottom-right) thus demonstrates that there is a relatively numerous population of reconnection sites far downstream of the shock, beyond $5 R_{E}$. We note that due to the short dwell times for this region, the statistical errors are large. Furthermore, given the the boundary between the transition region and the magnetosheath is not always clear, this downstream population may not be directly associated with the shock, and instead correspond to reconnection events observed within a turbulent magnetosheath.

The width of the distribution in $P\left(D_{s h}\right) / t_{\text {dwell }}$ suggests that the shock transition region which generates current sheets with electron outflows has a mean width of approximately $5 R_{E}$. However, we note that the width of the magnetosheath along the sub-solar point is expected to be of similar magnitude or less (Mejnertsen, Eastwood, Hietala, Schwartz, \& Chittenden, 2018). As discussed above, the width of the sheet-generating region is likely to be overestimated by the pseudo-distance measure.

Simulations of reconnection at high Mach number $\left(M_{A}>40\right)$, perpendicular shocks by Matsumoto et al. (2015) and Bohdan et al. (2017) show that current sheets and magnetic islands are generated upstream of the shock ramp, in the foot region. However, only $12 \%$ of reconnecting current sheets identified by the survey were observed within the upstream region $\left(t_{s h}<0\right)$. The relatively low fraction of upstream current sheets may reflect the differences in shock processes at the lower Mach numbers expected at Earth's bow shock. It may also represent an under-estimate due to the difficulty in defining a 

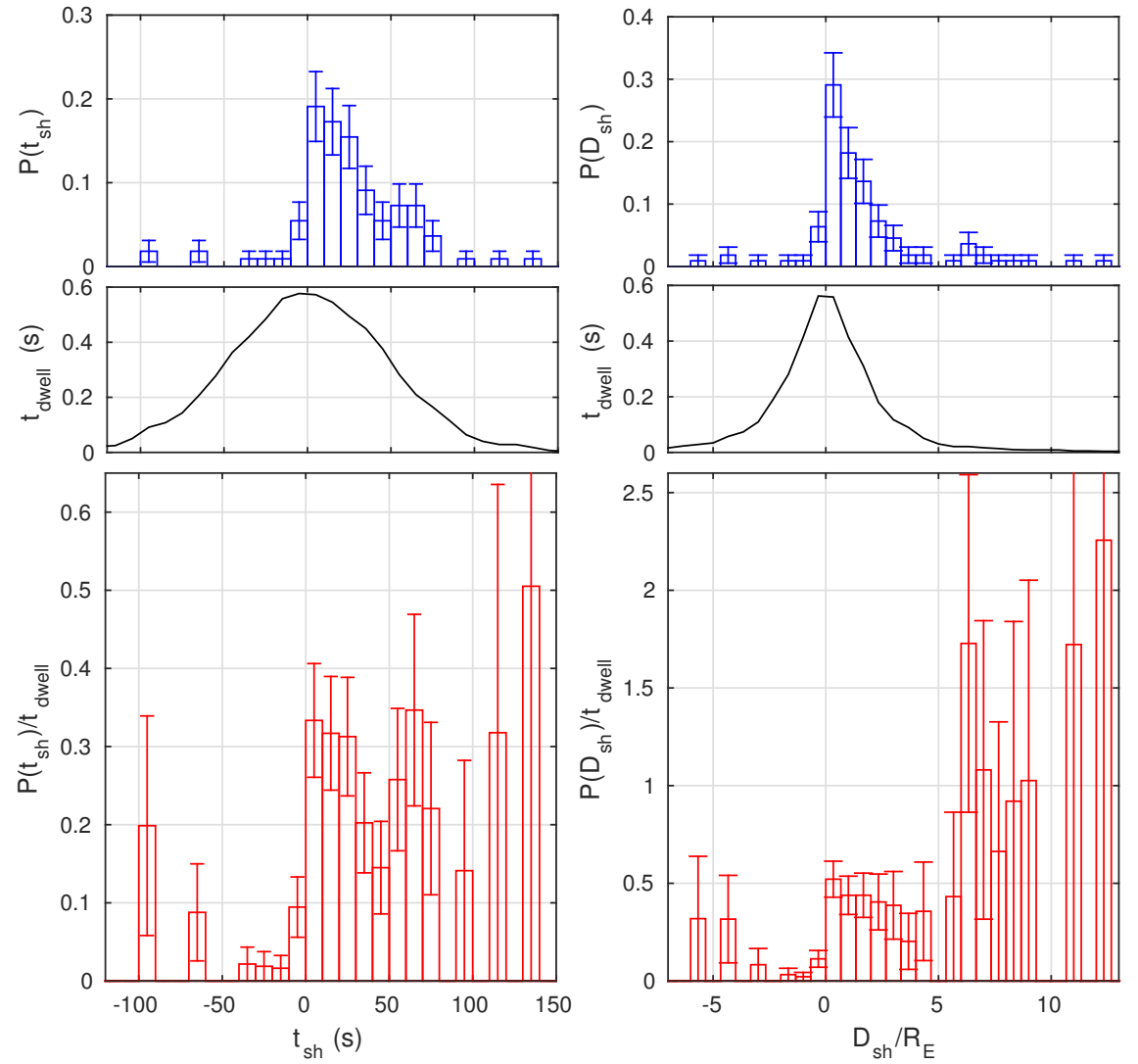

Figure 3. Top row: Histograms showing the probability distribution of the time (left) and distances (right) of each reconnection site from the shock ramp. The distances are given in units of Earth radii $R_{E}$. The times and distances are negative upstream in the solar wind, and positive downstream towards the magnetosheath. Middle: The dwell time of MMS1 at any given time and distance from the shock, across all available shock crossings included in the survey. Bottom: Probability distribution of the time and distance of reconnection sites from the shock, weighted by the inverse of the dwell time to account for selection biases. The error bars represent a $\sqrt{N}$ error, where $N$ is the number of events recorded within each bin. 
clear shock ramp for quasi-parallel shocks, and that some downstream events are likely associated with magnetosheath turbulence. However, the existence of a significant (or dominant) population of reconnecting current sheets downstream of the ramp is consistent with the hybrid simulations of quasi-parallel shocks reported by Gingell et al. (2017), for which instabilities in the foot generate magnetic islands that persist downstream of the ramp, in part due to the cyclic shock reformation cycle. The abundance of downstream current sheets observed in this survey suggests that the mechanism observed by Gingell et al. (2017) may generate more current sheets over all. This is not unexpected given that the Mach number of the simulations reported by Gingell et al. (2017) $\left(M_{A}=\right.$ 8 ) is more typical of Earth's bow shock.

\subsection{Sheet Properties}

In order to extract current sheet properties for each event selected by the survey, several fields and moments are fit to a Gaussian function $f_{i, \mathrm{fit}}(t)=\langle f\rangle+\Delta f \exp [-(t-$ $\left.\left.t_{c}\right)^{2} /\left(2 \sigma^{2}\right)\right]$, where $\langle f\rangle$ is the mean of the observed quantity for the given interval, and $\Delta f_{i}, t_{c}$ and $\sigma_{i}$ are free parameters corresponding to the peak height, peak centre and peak width respectively. The peak current density from the curlometer method $J_{\mathrm{fgm}}$ is determined from the height of the Gaussian fit to the medium variance component of the current density, $J_{M}$. The spatial width of the sheet is given by $L=\sigma_{J_{M}}\left\langle V_{e, N}\right\rangle$, where $\left\langle V_{e, N}\right\rangle$ is the mean of the normal component of the electron velocity across the single event interval, and $\sigma_{J_{M}}$ is the width of the Gaussian fit to the current density $J_{M}$. We therefore assume the validity of the Taylor hypothesis in determining current sheet width, i.e. the current sheets do not evolve significantly during the period over which they pass over the spacecraft. The speed of the electron jet and any observed ion jet are determined by the height of the Gaussian fits to the $L$-component of the bulk fields, given by $\Delta V_{e, L}$ and $\Delta V_{i, L}$ respectively.

Distributions and correlations of current sheet properties are shown in Figure 4. Each panel includes two single-variable histograms and a bivariate histogram to examine the correlation between two sheet properties. We can immediately determine from both the correlation coefficients $r$, and from the associated scatter plots, that correlations between current sheet properties are very weak. However, we note that this may be a result of the peak fitting associated with an automated survey; the errors in sheet properties are likely to be higher than for a manual treatment of each structure. Fur- 
thermore, since the Taylor hypothesis may not be valid in some cases, especially for those within a turbulent medium, the distribution of the current sheet width at the smallest scales may be distorted.

The lack of correlation between current sheet width $L$ and distance from the shock $D_{s h}$ (panel (f)) indicates that widening of current sheets does not occur as these structures are convected towards the magnetosheath, though the corresponding signatures of active reconnection may not be detectable for sheets at large scales. Similarly, the lack of correlation between $D_{s h}$ and either the peak current density $J_{f g m}$ (panel (d)) or the electron jet speed $V_{e L}$ (panel (a)) may indicate that the shock generates a diverse population of current sheets at the transition region within a short period of time, rather than generating the current sheets at a particular scale within a narrow layer. For example, the hybrid simulations presented by Gingell et al. (2017) show that current sheets and magnetic islands over multiple scales can be generated over a transition region spanning several ion inertial lengths, during a period less than the ion cyclotron time.

Panel (g) of Figure 4 shows the distribution of electron and ion jet speeds for the observed current sheets. In general, we find that the electron jets are significantly faster than their ion counterparts. For example, the mean electron jet speed is $1.4 V_{A}$, while the mean ion jet speed is $0.25 V_{A}$. The fastest electron jets are recorded at $5.5 V_{A}$, while the fastest ion jets are recorded at only $1.5 V_{A}$. Together with the low correlation coefficient, these results suggest that these current sheets strongly favour acceleration of electrons over ions. This is consistent with the observation of electron-only reconnection reported in the shock by Gingell et al. (2019); Wang et al. (2019), and in the magnetosheath by Phan et al. (2018). However, we note that electron-only reconnection in the sheath was observed for thinner current sheets with faster jets than in the shock transition region.

\subsubsection{Guide Field}

The statistics of current sheet guide field angle are shown in Figure 5. Here, the guide field angle is estimated using the equation $\theta_{\text {guide }}=\tan ^{-1}\left(B_{L 1} /\left\langle B_{M}\right\rangle\right)+\tan ^{-1}\left(B_{L 2} /\left\langle B_{M}\right\rangle\right)$, where $\left\langle B_{M}\right\rangle$ is the mean of the intermediate variance component of the magnetic field across the current carrying region, and $B_{L 1,2}$ are the maximum variable components of the magnetic field at the leading and trailing edge of the current carrying region. For 

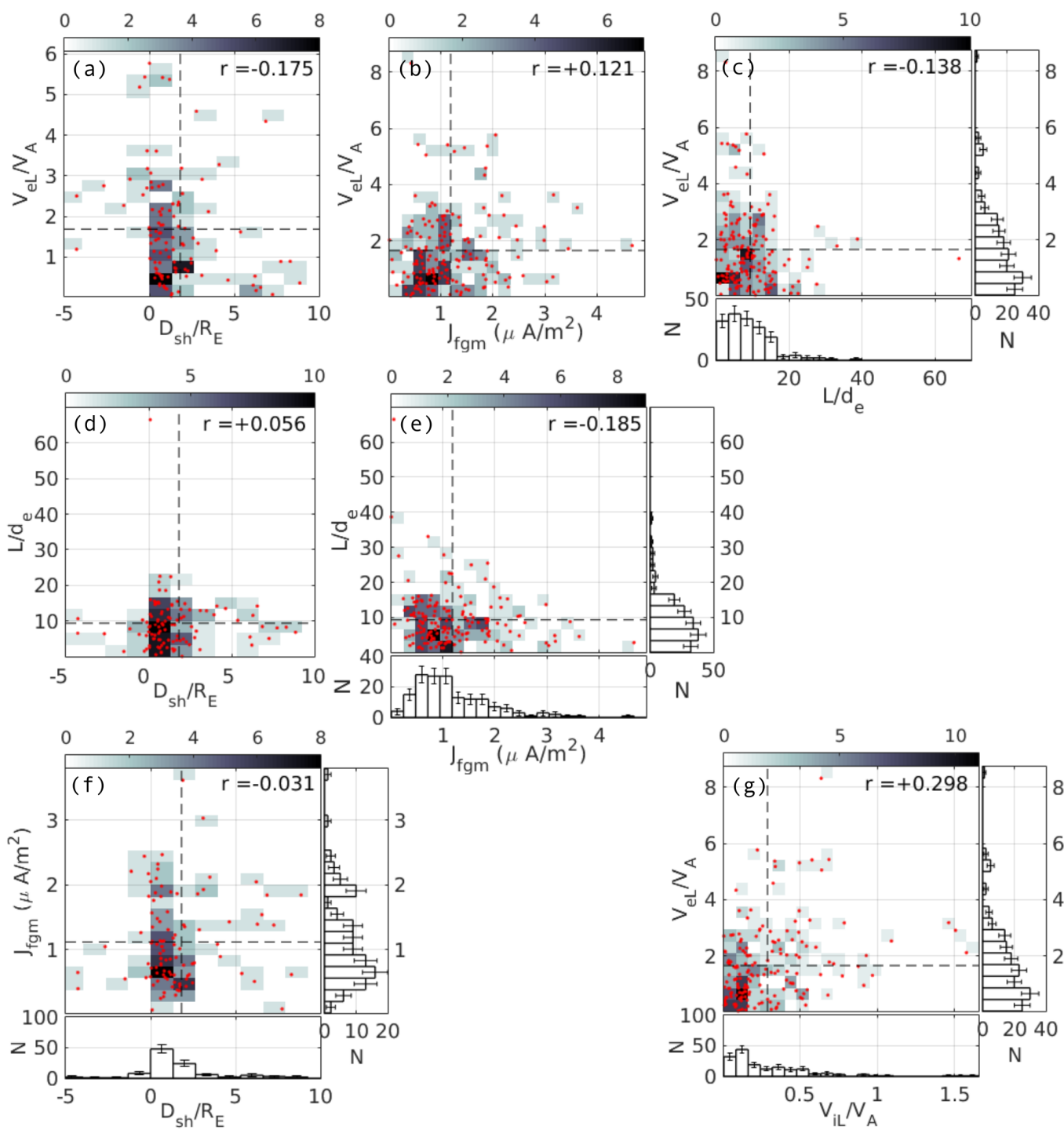

Figure 4. Scatter plots of reconnection site properties for all combinations of the electron jet speed $V_{e L}$ (top row), the current sheet width $L / d_{e}$ (middle row, right column), the peak current density from the curlometer method $J_{f g m}$ (bottom row, middle column), and the pseudo-distance of the current sheet from the shock ramp $D_{\text {sh }}$ (left column). Histograms of each quantity are also given at the end of each respective row and column. Each scatter plot is overlaid on a $2 \mathrm{D}$ histogram of the same data, with the number in each given bin displayed in the colour bar above each panel. Black dashed lines in each scatter represent the means. The correlation coefficient $r$ is also given for each pair of current sheet properties. The error bars represent a $\sqrt{N}$ error, where $N$ is the number of events recorded within each bin. Note that the dataset for the plots in the left column is reduced to only those for which the shock ramp timing analysis returned valid results. 

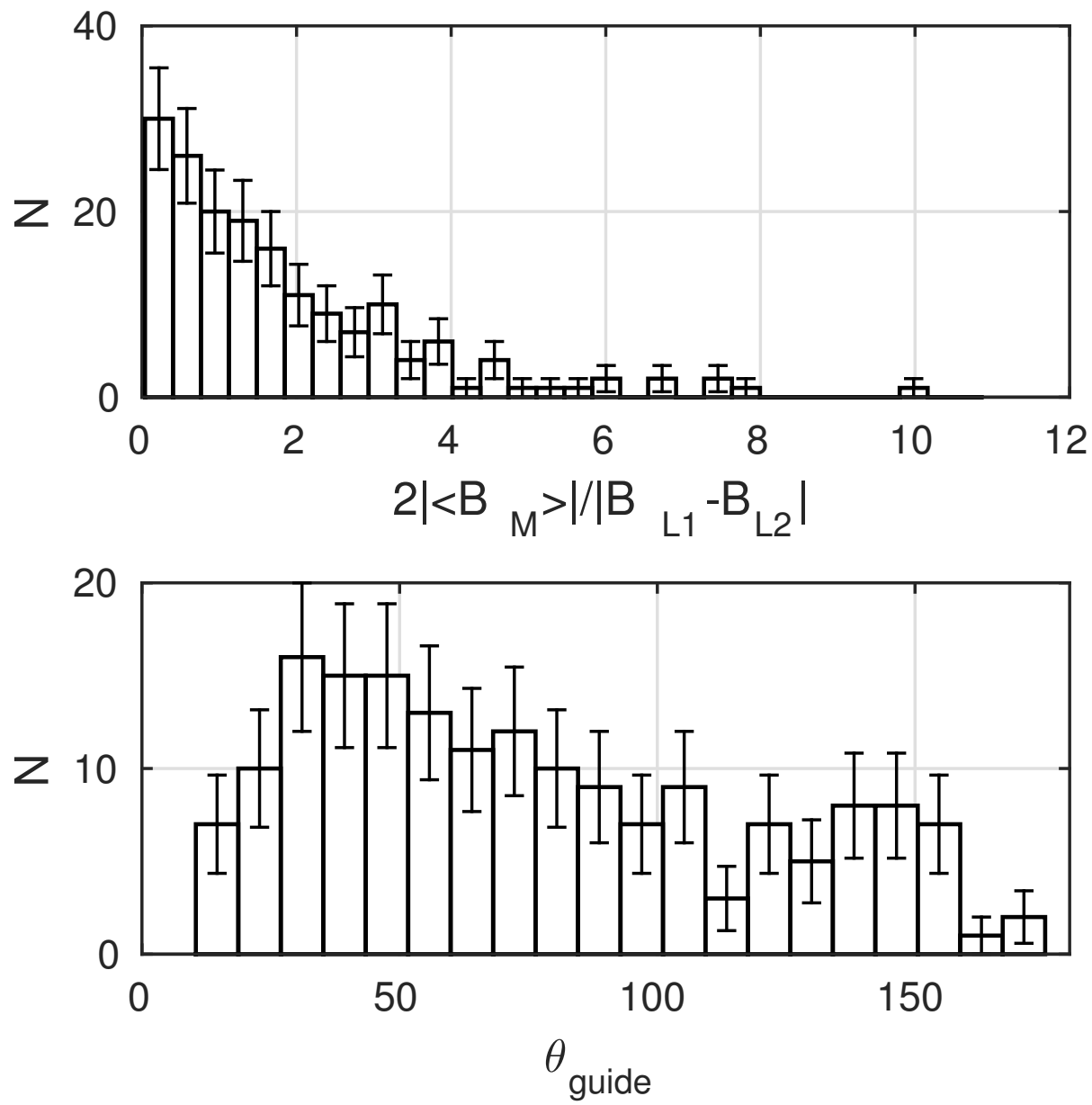

Figure 5. Histogram of the guide field angle $\theta_{\text {guide }}$ for all current sheets with electron outflow recorded by the survey. The error bars represent a $\sqrt{N}$ errors, where $N$ is the number of events recorded within each bin.

an anti-parallel current sheet with zero guide field, $\theta_{\text {guide }}=180^{\circ}$. If the guide field $B_{M}$ dominates, $\theta_{\text {guide }} \rightarrow 0$. The resulting distribution demonstrates that a broad range of guide field angles are observed for reconnecting current sheets. This is consistent with the generation of a broad geometry of structures from a turbulent or disordered region, rather than generation of current sheets from a coherent, highly-ordered instability that occurs for a favoured geometry. However, near anti-parallel current sheets with large guide field angles $\theta_{\text {guide }}>90^{\circ}$ are slightly less common. 


\subsubsection{Flow Structure}

In the classical picture of a reconnecting current sheet, the observation of a strong unipolar signature in the electron (or ion) velocity in the maximum variance direction suggests that a spacecraft has crossed an outflow or jet associated with active reconnection. However, for many of the events included in this analysis we observe significant electron flows that are bipolar or tripolar. Examples of each kind of structure are shown in Figure 6. Of the events identified by the survey, $53 \%$ have unipolar electron jets, $38 \%$ are bipolar, and the remaining $9 \%$ are tripolar. A bipolar structure in the bulk electron velocity may indicate an observation of field-aligned electron flow towards the $\mathrm{x}$-line on the other side of the separatrix from the jet (Eastwood et al., 2018; Øieroset et al., 2016; Phan et al., 2018; Wang et al., 2019), or in the most serendipitous cases the spacecraft may be observing oppositely directed outflow jets on crossing an electron diffusion region. A tripolar structure may indicate observation of field-aligned electron flow towards the $\mathrm{x}$-line on opposite sides of the Hall scale reconnection region. It is also important to recognise these variations in current sheet and flow structure may instead be a feature of current sheets associated with the disordered transition region of shock waves, or even with complex motion of the $\mathrm{x}$-line relative to the spacecraft. Unusual structures may also appear in cases for which the Taylor hypothesis is invalid, i.e. during the period over which the spacecraft traverses the jet, there is significant temporal evolution of the current sheet or a background turbulent medium. Hence, careful comparison to observations of turbulent reconnection in the solar wind and magnetosheath will be important for characterising these structures in future studies.

\subsubsection{Heating E Inflow Energy}

In order to quantify the heating occurring during shock reconnection events, we must examine the distributions of localised changes in the electron and ion temperatures as in Figure 7. Given the significant fluctuations of the temperature moments in the shock transition region, a simple Gaussian fit to the time series is not reliable. Instead, we first perform a 1s wide boxcar zero-phase digital filter to de-trend the data. The peak temperature changes $\Delta T_{e, i}$ are then determined by fitting a Gaussian function to the de-trended data, and extracting the height. 


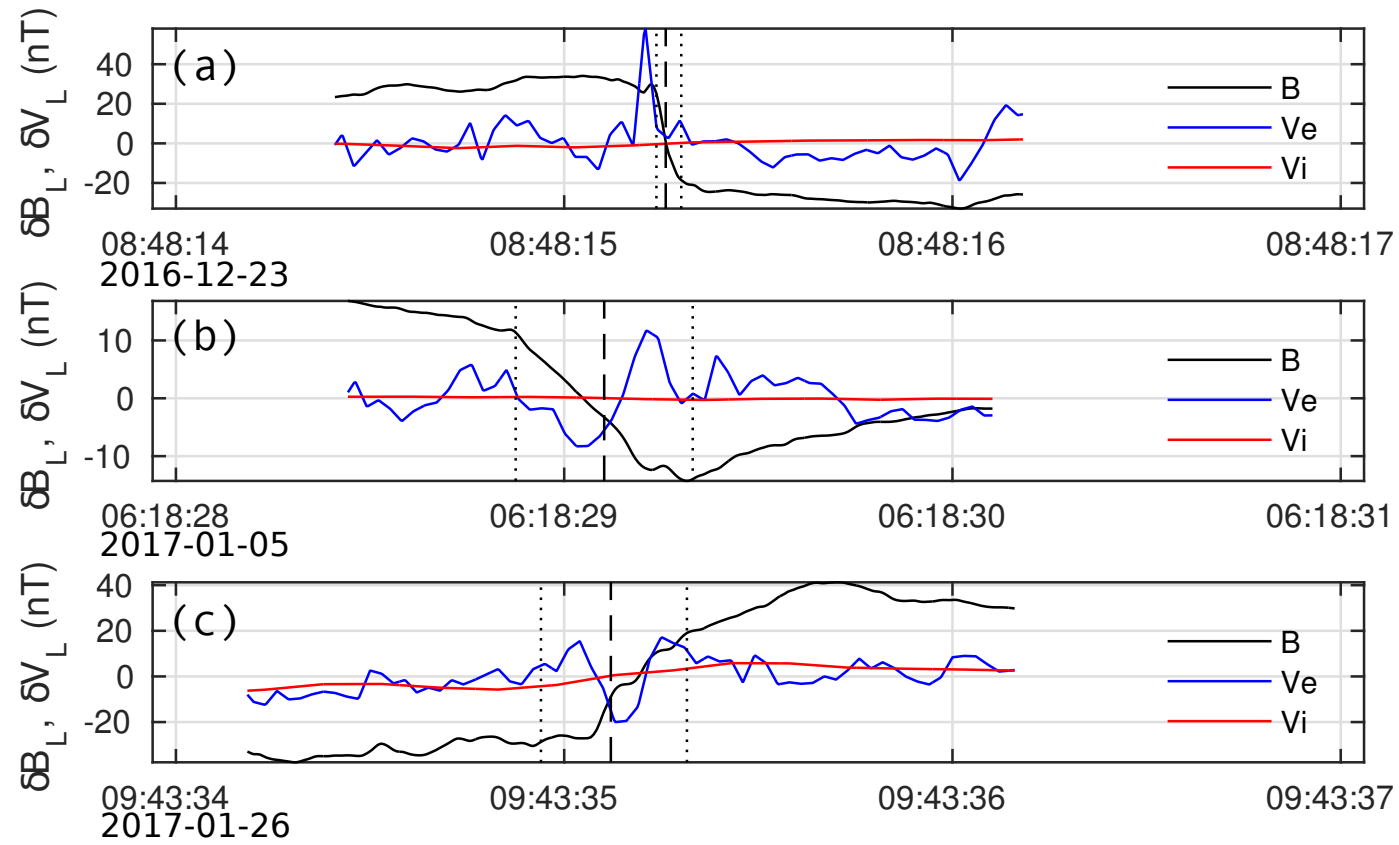

Figure 6. Structure of electron outflows on passing three current sheets identified by the survey. Time series show fluctuations of the maximum variance component of the magnetic field (black), electron bulk velocity (blue) and ion bulk velocity (red). Panel (a) shows a strong unipolar electron jet, panel (b) shows bipolar electron flows, and panel (c) shows tripolar electron flows in the current carrying region, bounded by vertical dotted lines. 

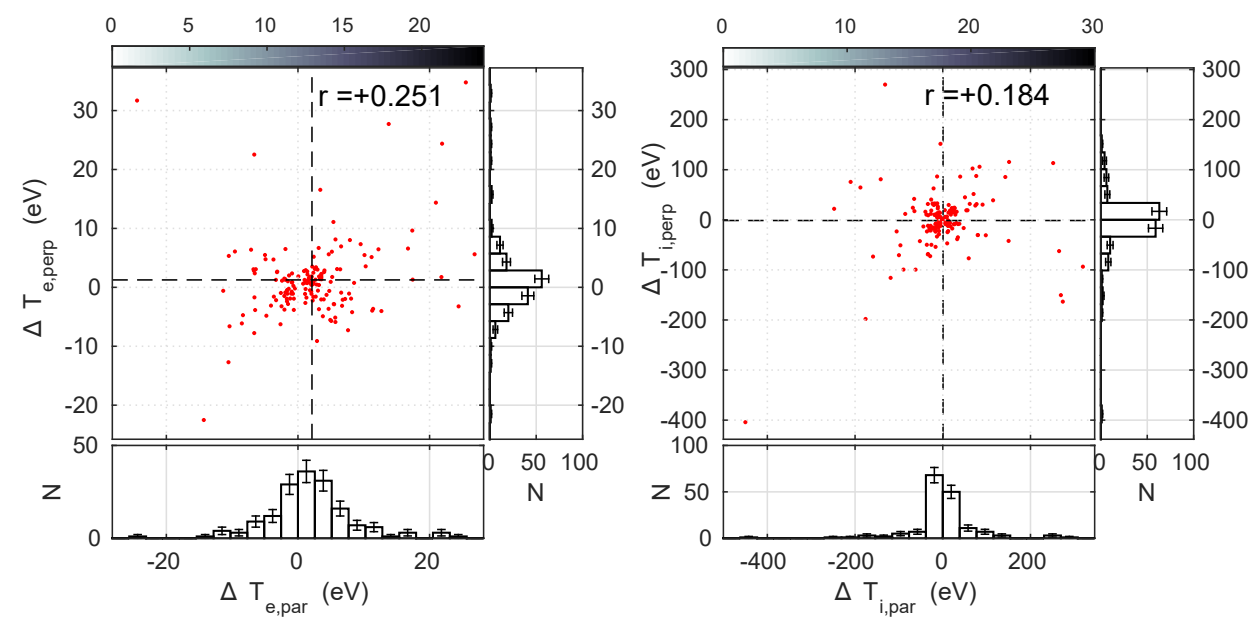

Figure 7. Scatter plots (red) and 2D histograms (grey) of the peak temperature change $\Delta T$, showing relationships between (a) parallel and perpendicular electron temperature changes. As in Figure 4, we also include 1D histograms of each quantity, and overlay dashed lines representing the means. The correlation coefficients $r$ are given in the top right of each panel. The error bars represent a $\sqrt{N}$ error, where $N$ is the number of events recorded within each bin.

In examining the electron response in Panel (a), we find that both the parallel and perpendicular temperatures have positive means, with $\Delta T_{e} \approx 2 e V$. However, the mean of the heating in each component is less than the width of the respective distributions, indicating that many events appear to cool. Additionally, extreme events appear to favour isotropic heating for which $\Delta T_{e, p a r} \approx \Delta T_{e, p e r p}$. Similar isotropy is seen for the ions in panel (b), and indeed the correlation coefficient for $\Delta T_{i, p a r}$ and $\Delta T_{i, p e r p}$ is largest among those shown in the paper. However there is no clear bias towards heating. Indeed, the mean perpendicular temperature chance $\Delta T_{e, p e r p}$ is negative. This may be representative of the electron-only coupling previously observed at shock reconnection sites (Gingell et al., 2019; Wang et al., 2019). We note that the width of these distributions may be indicative of the difficulty in fitting peaks across regions with significant inhomogeneity in the background.

In evaluating the heating across a current sheet, it is most instructive to compare the mean temperature change across the sheet with the magnetic inflow energy, $m_{i} V_{A L, \text { inflow }}^{2}$. The asymmetric inflow Alfvén speed energy for each potential current sheet is given by $V_{A L, \text { inflow }}=\frac{1}{2}\left[B_{1} B_{2}\left(B_{1}+B_{2}\right) / m_{p} \mu 0\left(n_{1} B_{2}+n_{2} B_{1}\right)\right]($ Cassak \& Shay, 2007; Swisdak \& Drake, 2007). Magnetic field and number densities $B_{1,2}$ and $n_{1,2}$ are taken at the edges 
of the current carrying region, where the magnitude of the current density has reduced to half its peak value.

Surveys of magnetic reconnection at the magnetopause have shown that the change in the mean electron temperature is given by $\delta T_{e} \sim 0.017 m_{i} V_{A L, \text { inflow }}^{2}$ (Phan et al., 2013), and the change in the mean ion temperature is given by $\delta T_{e} \sim 0.13 m_{i} V_{A L, \text { inflow }}^{2}$ (Phan et al., 2014). Given that this amount of heating was also observed for the shock reconnection event described by Gingell et al. (2019), it is reasonable that this trend might be observed in the histograms of the ratio $\delta T / m_{i} V_{A L, \text { inflow }}^{2}$ shown in Figure 8. However, for both electrons and ions the expected ratios 0.017 and 0.13 respectively are much smaller than the width of the distribution. This is probably because fluctuations associated with the inhomogeneous structure of the transition region are generally much larger than the expected temperature changes for the observed magnetic inflow energies. Indeed, in many of the events $\delta T$ even exceeds the magnetic inflow energy. We are therefore unable to extract a useful comparison of the bulk particle heating observed at the magnetopause to that observed at current sheets embedded in the shock transition region.

\section{Conclusions}

An automated survey of Magnetospheric Multiscale's burst mode data has been used to identify and characterise more than one hundred current sheets with electron outflows associated with the Earth's bow shock. These electron outflows are indicative of active reconnection occurring within the shock foot and the transition region extending downstream of the shock. However, we note that for this study we do not limit the search to only those events which show evidence of crossing the reconnection diffusion region. The survey demonstrates that at least one current sheet with electron outflow is observed by MMS for approximately $40 \%$ of shocks. These observations are found to occur across the full range of shock orientations $\theta_{B n}$ and Alfvén Mach numbers $M_{A}$, suggesting that reconnection is a universal process in shocks. However, analysis of the distribution of shock parameters among those that exhibit current sheets with electron flows, as compared to the distribution of all observed shocks, shows that quasi-parallel and high Mach number shocks may generate slightly more reconnecting current sheets than quasi-perpendicular and low Mach number shocks. This implies that while reconnection at shocks is not solely driven by phenomena that are more strongly associated with a given range of shock geometries (or Mach numbers), such as SLAMS in the quasi-parallel case (Schwartz et al., 

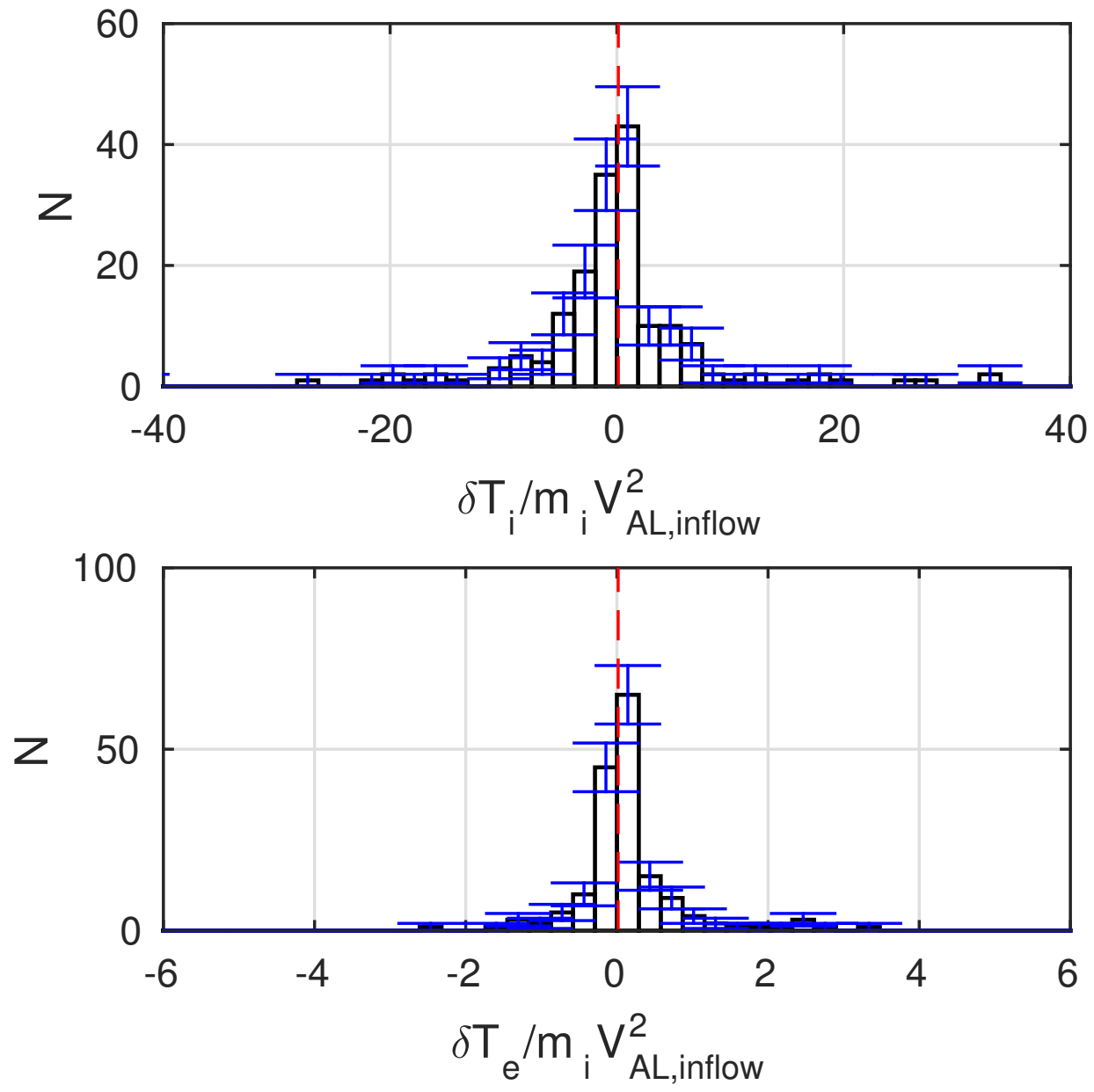

Figure 8. Histograms of the ratio of the mean temperature change across the current carrying region, $\delta T_{\text {tot }}=\delta\left(T_{\text {par }}+2 T_{\text {perp }}\right) / 3$, and the inflow energy $m_{i} V_{A, \text { inflow }}^{2}$ shown for both ions (top) and electrons (bottom). Red lines represent the expected ratios for magnetopause reconnection. The error bars represent a $\sqrt{N}$ error, where $N$ is the number of events recorded within each bin. 
1992), these mechanisms may enhance the generation of current sheets. Furthermore, given that quasi-parallel shocks generally have stronger fluctuations and turbulent structures than quasi-perpendicular shocks, it can be more difficult to identify reconnection sites embedded within the inhomogeneous medium. For that reason, the slight bias towards quasi-parallel shock observed within the collected dataset may be an underestimate, i.e. a more significant bias is likely.

Analysis of the location of active reconnection sites associated with shock waves has shown that the phenomenon is localised to the shock, and thus separated from turbulent reconnection occurring in the magnetosheath (Phan et al., 2018; Stawarz et al., 2019). This is consistent with the expectations set by hybrid simulations of Earth's bow shock presented by Gingell et al. (2017), which show that reconnection sites are generated on sub-ion timescales in a transient, localised transition region. However, it is yet unclear whether magnetosheath reconnection occurs via similar processes, i.e. generation of relaxing, coherent structures within a relatively narrow band of scales, or whether it is the end point of an active turbulent cascade. Indeed, current sheets in magnetosheath observations reported by Phan et al. (2018) are much thinner and with faster electron jets than those found by this survey (see Figure 4(c)). Given that only $12 \%$ of reconnecting current sheets identified by the survey are observed upstream of the shock ramp, and that observation of reconnection is more common at quasi-parallel shocks, we are able to conclude that the mechanism for generation of current sheets seen in high Mach number $\left(M_{A}>40\right)$, perpendicular simulations by Matsumoto et al. (2015) and Bohdan et al. (2017) (i.e. via turbulence generated by the ion Weibel instability within the shock foot), is unlikely to dominate across the shock parameter space observed at Earth's bow shock.

The survey presented here appears to favour current sheets which couple preferentially to the electrons, exhibiting relatively weak ion jets and ion heating. This is consistent with earlier observations of individual reconnection events in the shock (Gingell et al., 2019; Wang et al., 2019), and in the magnetosheath (Phan et al., 2018). Fully kinetic particle-in-cell simulations have shown that electron-only reconnection can occur for sufficiently small current sheets (Sharma Pyakurel et al., 2019). This further supports a model for which electron-only reconnection sites are generated at shocks on sub-ion timescales in a relatively narrow region of the transition layer. 
Given that temperature statistics are difficult to extract from the noise for most events, for both electrons and ions, we observe that the primary consequence of reconnection at shocks is with respect to the magnetic topology. That is, the complex connectivity of the magnetic field generated by instabilities in the shock foot and ramp is relaxed rapidly, within the disordered transition region which separates the shock region from the magnetosheath. Despite the difficulty in extracting trends in the temperature, we reiterate that some events do display localised heating commensurate with expectations set by observations of magnetopause reconnection, such as those reported by Gingell et al. (2019); Wang et al. (2019). Furthermore, Gingell et al. (2019) reported a gradual $7 \mathrm{eV}$ rise in the electron temperature across the transition region, which is not yet accounted for by the statistics presented here. This suggests that energy released by reconnection may be thermalised non-locally (though within the transition region) further complicating the process of extracting meaningful heating statistics within the disordered, inhomogeneous plasma.

In order more completely assess the integrated impact of magnetic reconnection on energy partition at collisionless shocks, we must still establish global trends in the structure of the transition region. For example, do shocks with more observations of current sheets with electron outflow exhibit a greater rise in the temperature across the transition region? We also seek a quantification of the density of reconnection sites within the shock transition region, given their frequency and three-dimensional extent.

\section{Acknowledgments}

This work was supported by the UK Science and Technology Facilities Council (STFC) grant ST/N000692/1. Data used in this research is publicly available at the MMS Science Data Center at the Laboratory for Atmospheric and Space Physics (LASP) hosted by the University of Colorado, Boulder (https://lasp.colorado.edu/mms/sdc/public/). IG was in part supported by the Royal Society University Research Fellowship URF $\backslash$ R1 $\backslash 191547$. Part of SJSs contributions are based upon work supported by the National Aeronautics and Space Administration under Grant MAG18_2-0071 issued through the HSR Program. Work at LASP was funded by the NASA MMS project, and work at IRAP was supported by CNRS and CNES. We also acknowledge the International Space Science Institute (ISSI) International Teams programme for providing collaborative opportunity for this work. 


\section{References}

Abraham-Shrauner, B. (1972). Determination of magnetohydrodynamic shock normals. Journal of Geophysical Research, 77, 736. doi: 10.1029/ JA077i004p00736

Auer, P. L., Hurwitz, H., Jr., \& Kilb, R. W. (1962, March). Large-Amplitude Magnetic Compression of a Collision-Free Plasma. II. Development of a Thermalized Plasma. Physics of Fluids, 5, 298-316. doi: 10.1063/1.1706615

Biskamp, D., \& Welter, H. (1972). Structure of the Earth's bow shock. Journal of Geophysical Research, 77, 6052. doi: 10.1029/JA077i031p06052

Bohdan, A., Niemiec, J., Kobzar, O., \& Pohl, M. (2017, September). Electron Preacceleration at Nonrelativistic High-Mach-number Perpendicular Shocks. The Astrophysical Journal, 847, 71. doi: 10.3847/1538-4357/aa872a

Burch, J. L., Torbert, R. B., Phan, T. D., Chen, L.-J., Moore, T. E., Ergun, R. E., ... Chandler, M. (2016, June). Electron-scale measurements of magnetic reconnection in space. Science, 352, aaf2939. doi: 10.1126/science.aaf2939

Burgess, D. (1989, May). Cyclic behavior at quasi-parallel collisionless shocks. Geophysical Research Letters, 16, 345-348. doi: 10.1029/GL016i005p00345

Burgess, D. (1995). Foreshock-shock interaction at collisionless quasi-parallel shocks. Advances in Space Research, 15, 159-169. doi: 10.1016/0273-1177(94)00098-L

Burgess, D., \& Scholer, M. (2015). Collisionless Shocks in Space Plasmas. Cambridge University Press.

Cassak, P. A., \& Shay, M. A. (2007). Scaling of asymmetric magnetic reconnection: General theory and collisional simulations. Physics of Plasmas, 14(10), 102114. Retrieved from https://doi.org/10.1063/1.2795630 doi: 10.1063/ 1.2795630

Chasapis, A., Yang, Y., Matthaeus, W. H., Parashar, T. N., Haggerty, C. C., Burch, J. L., ... Russell, C. T. (2018, Jul). Energy Conversion and Collisionless Plasma Dissipation Channels in the Turbulent Magnetosheath Observed by the Magnetospheric Multiscale Mission. The Astrophysical Journal, 862(1), 32. doi: $10.3847 / 1538-4357 /$ aac775

Eastwood, J. P., Mistry, R., Phan, T. D., Schwartz, S. J., Ergun, R. E., Drake, J. F., ... Russell, C. T. (2018). Guide field reconnection: Exhaust structure and heating. Geophysical Research Letters, 45(10), 4569-4577. Retrieved 
from https://agupubs.onlinelibrary.wiley.com/doi/abs/10.1029/ 2018GL077670 doi: 10.1029/2018GL077670

Gingell, I., Schwartz, S. J., Burgess, D., Johlander, A., Russell, C. T., Burch, J. L., ... Wilder, F. (2017, November). MMS Observations and Hybrid Simulations of Surface Ripples at a Marginally Quasi-Parallel Shock. Journal of Geophysical Research (Space Physics), 122, 11. doi: 10.1002/2017JA024538

Gingell, I., Schwartz, S. J., Eastwood, J. P., Burch, J. L., Ergun, R. E., Fuselier, S., .. Wilder, F. (2019, February). Observations of Magnetic Reconnection in the Transition Region of Quasi-Parallel Shocks. Geophys. Res. Lett., 46, 1177-1184. doi: 10.1029/2018GL081804

Gosling, J. T., \& Phan, T. D. (2013, February). Magnetic Reconnection in the Solar Wind at Current Sheets Associated with Extremely Small Field Shear Angles. Astrophysical Journal Letters, 763, L39. doi: 10.1088/2041-8205/763/2/L39

Gosling, J. T., \& Robson, A. E. (1985). Ion reflection, gyration, and dissipation at supercritical shocks. Washington DC American Geophysical Union Geophysical Monograph Series, 35, 141-152. doi: 10.1029/GM035p0141

Gosling, J. T., Skoug, R. M., McComas, D. J., \& Smith, C. W.

(2005, January). Direct evidence for magnetic reconnection in the solar wind near 1 AU. Journal of Geophysical Research (Space Physics), 110, A01107. doi: 10.1029/2004JA010809

Hada, T., Oonishi, M., Lembège, B., \& Savoini, P. (2003, June). Shock front nonstationarity of supercritical perpendicular shocks. Journal of Geophysical Research (Space Physics), 108, 1233. doi: 10.1029/2002JA009339

Krauss-Varban, D., \& Omidi, N. (1991, October). Structure of medium Mach number quasi-parallel shocks - Upstream and downstream waves. Journal of Geophysical Research, 96, 17. doi: 10.1029/91JA01545

Lindqvist, P.-A., Olsson, G., Torbert, R. B., King, B., Granoff, M., Rau, D., ... Tucker, S. (2016, Mar 01). The spin-plane double probe electric field instrument for mms. Space Science Reviews, 199(1), 137-165. Retrieved from https://doi.org/10.1007/s11214-014-0116-9 doi: 10.1007/s11214-014-0116-9

Matsumoto, Y., Amano, T., Kato, T. N., \& Hoshino, M. (2015). Stochastic electron acceleration during spontaneous turbulent reconnection in a strong shock wave. 
Science, 347(6225), 974-978. Retrieved from http://science.sciencemag .org/content/347/6225/974 doi: 10.1126/science.1260168

Matthaeus, W. H., \& Lamkin, S. L. (1986). Turbulent magnetic reconnection. The Physics of Fluids, 29(8), 2513-2534. Retrieved from https://aip.scitation .org/doi/abs/10.1063/1.866004 doi: 10.1063/1.866004

Mejnertsen, L., Eastwood, J. P., Hietala, H., Schwartz, S. J., \& Chittenden, J. P. $(2018,1)$. Global mhd simulations of the earth's bow shock shape and motion under variable solar wind conditions. Journal of Geophysical Research: Space Physics, 123(1), 259-271. Retrieved from https://doi.org/10.1002/ 2017JA024690 doi: 10.1002/2017JA024690

Morse, D. L., Destler, W. W., \& Auer, P. L. (1972, January). Nonstationary Behavior of Collisionless Shocks. Physical Review Letters, 28, 13-16. doi: 10.1103/ PhysRevLett.28.13

Øieroset, M., Phan, T. D., Haggerty, C., Shay, M. A., Eastwood, J. P., Gershman, D. J., ... Malakit, K. (2016). Mms observations of large guide field symmetric reconnection between colliding reconnection jets at the center of a magnetic flux rope at the magnetopause. Geophysical Research Letters, 43(11), 55365544. Retrieved from https://agupubs.onlinelibrary.wiley.com/doi/abs/ 10.1002/2016GL069166 doi: 10.1002/2016GL069166

Øieroset, M., Phan, T. D., Shay, M. A., Haggerty, C. C., Fujimoto, M., Angelopoulos, V., ... Mozer, F. S. (2017). Themis multispacecraft observations of a reconnecting magnetosheath current sheet with symmetric boundary conditions and a large guide field. Geophysical Research Letters, 44(15), 7598-7606. Retrieved from https://agupubs.onlinelibrary.wiley.com/doi/abs/ 10.1002/2017GL074196 doi: 10.1002/2017GL074196

Paschmann, G., Papamastorakis, I., Sckopke, N., Haerendel, G., Sonnerup, B. U. O., Bame, S. J., ... Elphic, R. C. (1979, November). Plasma acceleration at the earth's magnetopause - Evidence for reconnection. Nature, 282, 243-246. doi: $10.1038 / 282243 \mathrm{a} 0$

Peredo, M., Slavin, J. A., Mazur, E., \& Curtis, S. A. ～(1995, May). Threedimensional position and shape of the bow shock and their variation with Alfvenic, sonic and magnetosonic Mach numbers and interplanetary magnetic field orientation. Journal of Geophysical Research, 100, 7907-7916. doi: 
10.1029/94JA02545

Phan, T. D., Drake, J. F., Shay, M. A., Gosling, J. T., Paschmann, G., Eastwood, J. P., ... Angelopoulos, V. (2014, October). Ion bulk heating in magnetic reconnection exhausts at Earth's magnetopause: Dependence on the inflow Alfvén speed and magnetic shear angle. Geophys. Res. Lett., 41, 7002-7010. doi: 10.1002/2014GL061547

Phan, T. D., Eastwood, J. P., Shay, M. A., Drake, J. F., Sonnerup, B. U. Ö., Fujimoto, M., ... Magnes, W. (2018, May). Electron magnetic reconnection without ion coupling in Earth's turbulent magnetosheath. Nature, 557, 202206. doi: 10.1038/s41586-018-0091-5

Phan, T. D., Kistler, L. M., Klecker, B., Haerendel, G., Paschmann, G., Sonnerup, B. U. Ö., ... Reme, H. (2000, April). Extended magnetic reconnection at the Earth's magnetopause from detection of bi-directional jets. Nature, 404, 848-850. doi: $10.1038 / 35009050$

Phan, T. D., Shay, M. A., Gosling, J. T., Fujimoto, M., Drake, J. F., Paschmann, G., ... Angelopoulos, V. (2013, September). Electron bulk heating in magnetic reconnection at Earth's magnetopause: Dependence on the inflow Alfvén speed and magnetic shear. Geophysical Research Letters, 40, 4475-4480. doi: $10.1002 /$ grl.50917

Pollock, C., Moore, T., Jacques, A., Burch, J., Gliese, U., Saito, Y., ... Zeuch, M. (2016, March). Fast Plasma Investigation for Magnetospheric Multiscale. Space Science Reviews, 199, 331-406. doi: 10.1007/s11214-016-0245-4

Retinò, A., Sundkvist, D., Vaivads, A., Mozer, F., André, M., \& Owen, C. J. (2007, April). In situ evidence of magnetic reconnection in turbulent plasma. Nature Physics, 3, 236-238. doi: 10.1038/nphys574

Robert, P., Dunlop, M. W., Roux, A., \& Chanteur, G. (1998). Accuracy of Current Density Determination. ISSI Scientific Reports Series, 1, 395-418.

Russell, C. T., Anderson, B. J., Baumjohann, W., Bromund, K. R., Dearborn, D., Fischer, D., ... Richter, I. (2016, March). The Magnetospheric Multiscale Magnetometers. Space Science Reviews, 199, 189-256. doi $10.1007 / \mathrm{s} 11214-014-0057-3$

Scholer, M., Shinohara, I., \& Matsukiyo, S. (2003, January). Quasi-perpendicular shocks: Length scale of the cross-shock potential, shock reformation, and im- 
plication for shock surfing. Journal of Geophysical Research (Space Physics), 108, 1014. doi: 10.1029/2002JA009515

Schwartz, S. J. (1998). Analysis Methods for Multi-Spacecraft Data. ISSI Sci. Rep. Ser., 1, 249.

Schwartz, S. J., Burgess, D., Wilkinson, W. P., Kessel, R. L., Dunlop, M., \& Luehr, H. (1992, April). Observations of short large-amplitude magnetic structures at a quasi-parallel shock. Journal of Geophysical Research, 97, 4209-4227. doi: 10.1029/91JA02581

Servidio, S., Matthaeus, W. H., Shay, M. A., Cassak, P. A., \& Dmitruk, P. (2009, March). Magnetic Reconnection in Two-Dimensional Magnetohydrodynamic Turbulence. $\quad$ Physical Review Letters, 102(11), $115003 . \quad$ doi: 10.1103/PhysRevLett.102.115003

Sharma Pyakurel, P., Shay, M. A., Phan, T. D., Matthaeus, W. H., Drake, J. F., TenBarge, J. M., ... Chasapis, A. (2019, Jan). Transition from ion-coupled to electron-only reconnection: Basic physics and implications for plasma turbulence. arXiv e-prints, arXiv:1901.09484.

Stawarz, J. E., Eastwood, J. P., Phan, T. D., Gingell, I. L., Shay, M. A., Burch, J. L., ... Franci, L. (2019, June). Properties of the Turbulence Associated with Electron-only Magnetic Reconnection in Earth's Magnetosheath. Astrophysical Journal Letters, 877, L37. doi: 10.3847/2041-8213/ab21c8

Sundkvist, D., Retinò, A., Vaivads, A., \& Bale, S. D. (2007, July). Dissipation in Turbulent Plasma due to Reconnection in Thin Current Sheets. Physical Review Letters, $99(2)$, 025004. doi: 10.1103/PhysRevLett.99.025004

Swisdak, M., \& Drake, J. F. (2007). Orientation of the reconnection xline. Geophysical Research Letters, 34(11). Retrieved from https:// agupubs.onlinelibrary.wiley.com/doi/abs/10.1029/2007GL029815 doi: 10.1029/2007GL029815

Torbert, R. B., Russell, C. T., Magnes, W., Ergun, R. E., Lindqvist, P.-A., LeContel, O., ... Lappalainen, K. (2016, March). The FIELDS Instrument Suite on MMS: Scientific Objectives, Measurements, and Data Products. Space Science Reviews, 199, 105-135. doi: 10.1007/s11214-014-0109-8

Vasyliunas, V. M. (1975, February). Theoretical models of magnetic field line merging. I. Reviews of Geophysics and Space Physics, 13, 303-336. doi: 10.1029/ 
RG013i001p00303

Vörös, Z., Yordanova, E., Varsani, A., Genestreti, K. J., Khotyaintsev, Y. V., Li, W., ... Saito, Y. (2017). Mms observation of magnetic reconnection in the turbulent magnetosheath. Journal of Geophysical Research: Space Physics, 122(11), 11,442-11,467. Retrieved from https://agupubs.onlinelibrary .wiley.com/doi/abs/10.1002/2017JA024535 doi: 10.1002/2017JA024535

Wang, S., Chen, L.-J., Bessho, N., Hesse, M., Wilson, L. B., Giles, B., .. Burch, J. L. (2019, January). Observational Evidence of Magnetic Reconnection in the Terrestrial Bow Shock Transition Region. Geophys. Res. Lett., 46, 562-570. doi: $10.1029 / 2018$ GL080944

Yordanova, E., Vörös, Z., Varsani, A., Graham, D. B., Norgren, C., Khotyaintsev, Y. V., .. Saito, Y. (2016, Jun). Electron scale structures and magnetic reconnection signatures in the turbulent magnetosheath. Geophysical Research Letters , 43(12), 5969-5978. doi: 10.1002/2016GL069191 